\section{SMGrøup}

\section{SM Journal of Pediatric Surgery}

\section{Article Information}

Received date: Aug 24, 2015

Accepted date: Oct 12, 2015

Published date: Oct 30, 2015

\section{${ }^{*}$ Corresponding author}

Mohamed Jallouli, Department of pediatric surgery, HediChaker Teaching Hospital, 3029 Sfax, Tunisia, Tel: 00216 23209 111; Fax: 0021674241384; Email: mohamed.jallouli@rns.tn

Distributed under Creative Commons CC-BY 4.0

Keywords Renal failure; Children; Surgery

\title{
Surgical Aspect of Pediatric Peritoneal Dialysis: A Survey of the Maghreb Pediatric Surgeon
}

\author{
Mohamed Jallouli ${ }^{1 \star}$, Mahdi BenDhaou ${ }^{2}$ and Riadh Mhiri ${ }^{2}$ \\ ${ }^{1}$ Department of Pediatric Surgery, HediChaker Teaching Hospital, Tunisia \\ ${ }^{2}$ School of medicine, University of Sfax, Tunisia
}

\section{Abstract}

Objective: To assess the routine surgical practices of consultant pediatric surgeon in the Maghrebian country in their management of peritoneal dialysis.

Methods: During the twelve Maghrebiancongess of pediatric surgery in Tunis May. 2012, a session of quiz survey (4 Questions) about the surgical aspect of pediatric peritoneal dialysis was dedicated. 60 surgeons participate to this session. An electronic voting system was used.

Results: $60 \%$ of surgeons practice a mini-laparotomy for catheter insertion. Half of the participants use a double cuff catheter while the other half uses a simple cuff catheter. $65 \%$ of participant allows initiation of dialysis within 7 days post catheter insertion. Only $13 \%$ of the pediatric surgeons perform an omentectomy at the time of the insertion of the catheter.

Conclusion: The placement of a PD catheter requires an experienced surgeon and should be given appropriate priority. Adhesions to guidelines may improve outcome of patient by reducing the early peritoneal dialysis catheter failure in children in our countries.

\section{Introduction}

Peritoneal Dialysis (PD) is currently the therapy of choice to bridge pediatric patients with endstage renal disease to transplant. This technique allows the preservation of quality of life, residual renal function and vascular capital. The roles of the pediatric surgeon involve the insertion of the dialysis catheter and the management of some complications with pediatric nephrologists' collaborations. The introduction of peritoneal dialysis in children in the Maghreb countries is relatively recent and dates back to the 2000s. Despite the established use of this therapy, aspects regarding the technical placement of PD catheters remain no standardized. Few recommendations in the placement of peritoneal catheters could be made with any high grade of evidence or with pediatric-specific evidence toward a standard of care. Technical aspects of catheter placement. Such as the number of cuffs, catheter type and exit-site direction, are based on evidence from nonrandomized trials at best [1]. The aim of survey is to assess the routine surgical practices of consultant pediatric surgeon in the Maghreb region in their management of peritoneal dialysis and their accordance with guidelines.

\section{Methods}

During the twelve's Maghrebian congress of pediatric surgery in Tunis May. 2012, a session of quiz survey (4 Questions) about the surgical aspect of pediatric peritoneal dialysis was dedicated. The 4 questions were:

1. What's your technique of catheter placement?

2. Did you use single or double-cuff catheters?

3. Days did you advocate an early use of catheters (Within 7 days post catheter insertion)

4. Did you perform an omentectomy?

60 surgeons participate to this session. An electronic voting system using multi choice Keypad was anonymous. The results were displayed instantly

\section{Results}

$60 \%$ of surgeons practice a mini-laparotomy for catheter insertion, laparoscopy and percutaneous insertion represent respectively 11 and $15 \%$ (Figure 1). Half of the participants use a double cuff catheter while the other half uses a simple cuff catheterr the selection of participants was more related to the availability of one or the other type of the catheter (Figure 2). $65 \%$ of participant allows initiation of dialysis within 7 days post catheter insertion (Figure 3). Only 13\% of the pediatric surgeons perform an omentectomy at the time of the insertion of the catheter (Figure 4). 

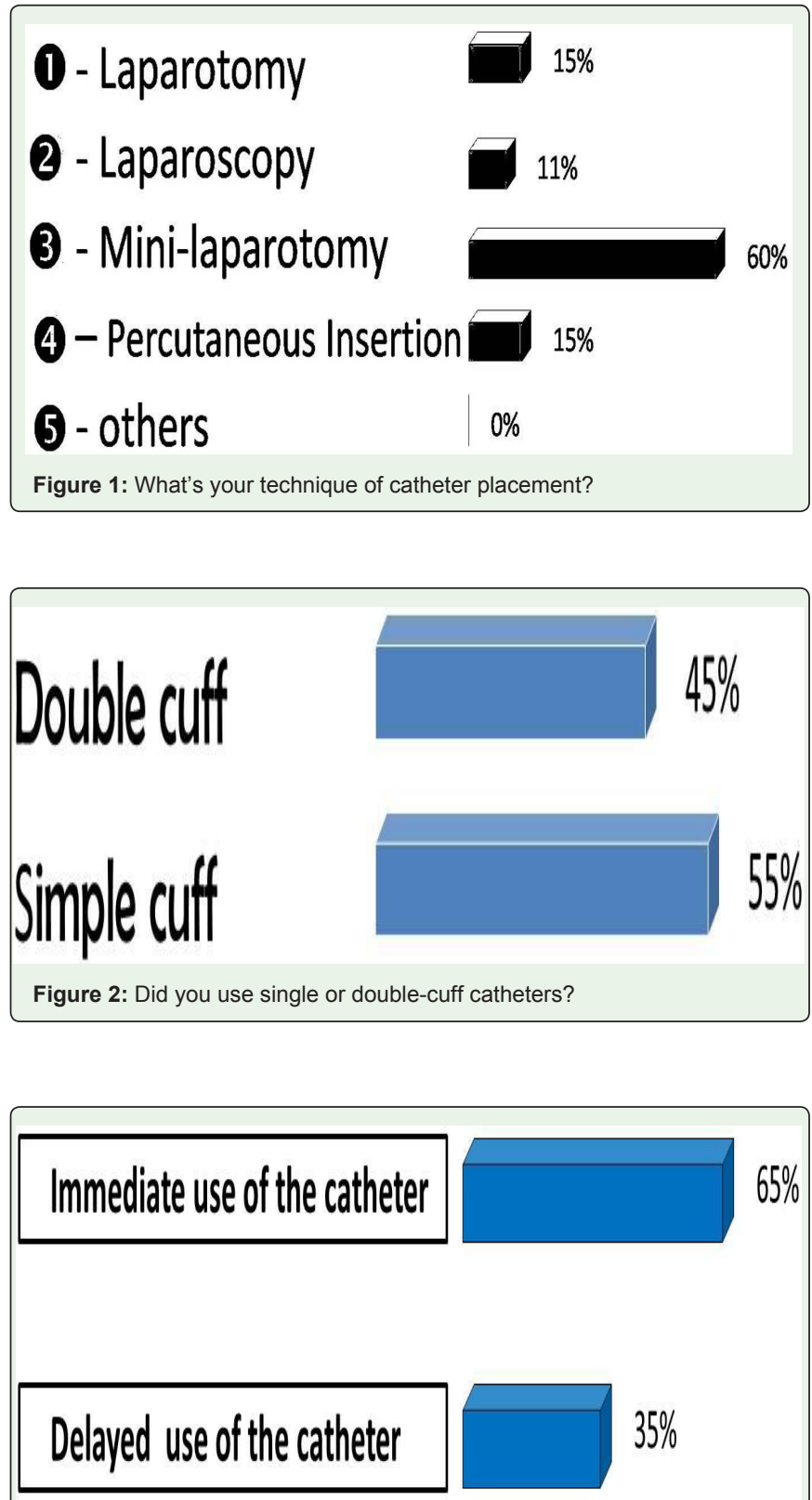

Figure 3: Days Did you advocate an early use of catheters (Within 7 days post catheter insertion).

\section{O Yes $13 \%$
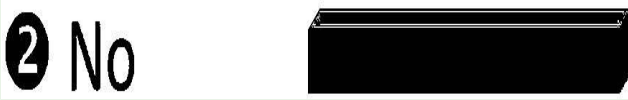 \\ $87 \%$}

Figure 4: Did you perform an omentectomy?

\section{Discussion}

Catheter placement first developed in 1968 [2], was thought to be the key to successful Peritoneal Dialysis (PD). In 2004, Washburn et al [3] published the results of asurvey of members of the American Pediatric Surgical Association and the Canadian Association of Pediatric Surgeons regarding surgical technique for PD catheter placement. Their results demonstrated significant variability in all aspects of surgical technique. These findings were also reflected in our survey. Two meta-analyses compare laparoscopy with open placement of peritoneal dialysis catheter [4,5]. Xie et $\mathrm{Al}$ found that there is no difference in catheter complication or failure rates between laparoscopic and open placements [5]. On the other side Hagen et al concluded that there is some evidence in favor of laparoscopic insertion technique for having a higher one year catheter survival and less migration, which would be clinically relevant [4]. This study does not include pediatric patient. Some pediatric nephrologists advocate the percutaneous placement of PDS, this technique seems to be reliable and safe and cost effective methods [6].

Concerning the use of single or double cuffed PDC; doublecuffed curled catheters are preferred in most children. A single-cuffed catheter may be needed in infants less than $3 \mathrm{~kg}$ [7]. Early and delayed use of PDC doesn't' affect the outcome [1]. Nikibakhsh et al showed in a cohort of 56 children's that catheter- related complications were not higher than those previously reported using urgently PD catheters when PD could be initiated immediately after catheter implantation [8].The Canadian Association of Pediatric Nephrologists recommend that timing for the initiation of dialysis post catheter insertion should be left to the discretion of the center recognizing the need for wound healing and If initiation of dialysis is required within 7 days postcatheter insertion, low volumes should be commenced [7].

Omentectomy is thought to reduce the risk of adhesions and catheter occlusion and improve catheter survival [9-11]. The obstruction is usually caused by omentum wrapping around the catheter and occluding the ports [7]. During the catheter insertion, the tip is placed deep into the pelvis to avoid the catheter being obstructed by the omentum. However, the peritoneal catheters are frequently blocked. Routine partial omentectomy at the time of catheter placement may help to prevent this complication [12]. Despite this consideration, there is no evidence in the pediatric literature to demonstrate any benefit of omentectomy, although there is some evidence in adults [7]. In our study, inserting by mini laparotomy, early use of catheter are explained in most cases by the emergency context of the peritoneal catheter insertion. Omentectomy is not advocate by the majority of the participants to this survey. A Maghrebian prospective study dealing with the surgical aspects of dialysis in children is necessary to substantiate these results.

\section{Conclusion}

The placement of a PD catheter requires an experienced surgeon and should be given appropriate priority. Adhesions to guidelines may improve outcome of patient by reducing the early peritoneal dialysis catheter failure in children in our countries.

\section{References}

1. Ladd AP, Breckler FD, Novotny NM. Impact of primary omentectomy on longevity of peritoneal dialysis catheters inchildren. Am J Surg. 2011; 201: 401-414. 
2. Tenckhoff $\mathrm{H}$, Schechter $\mathrm{H}$. A bacteriologically safe peritoneal access device. Trans Am Soc Artif Intern Organs. 1968; 14: 181-187.

3. Aksu N, Yavascan O, Anil M, Kara OD, Erdogan H. A ten-year singlecentre experience in children on chronic peritoneal dialysis--significance of percutaneous placement of peritoneal dialysis catheters. Nephrol Dial Transplant. 2007; 22: 2045-2051.

4. White CT, Gowrishankar M, Feber J, Yiu V, Canadian Association of Pediatric Nephrologists (CAPN), et al. Clinical practice guidelines for pediatric peritoneal dialysis. Pediatr Nephrol. 2006; 21: 1059-1066.

5. Cribbs RK, Greenbaum LA, Heiss KF. Risk factors for early peritoneal dialysis catheter failure in children. J Pediatr Surg. 2010; 45: 585-589.

6. Dönmez O, Durmaz O, Ediz B, Cigerdelen N, Kocak S. Catheter-related complications in children on chronic peritoneal dialysis. Adv Perit Dial. 2005 21: $200-203$

7. Hagen SM, Lafranca JA, Steyerberg EW, Äzermans JN, Dor FJ. Laparoscopic versus open peritoneal dialysis catheter insertion: a meta-analysis. PLoS One. 2013; 8: e56351.
8. Nikibakhsh AA, Mahmoodzadeh H, Vali M, Enashaei A, Asem A. Outcome of immediate use of the permanent peritoneal dialysis catheter in children with acute and chronic renal failure. Iran J Pediatr. 2013; 23: 171-176.

9. Phan J, Stanford S, Zaritsky JJ, DeUgarte DA. Risk factors for morbidity and mortality in pediatric patients with peritoneal dialysis catheters. J Pediatr Surg. 2013; 48: 197-202.

10. Washburn KK, Currier H, Salter KJ, Brandt ML. Surgical technique for peritoneal dialysis catheter placement in the pediatric patient: a North American survey. Adv Perit Dial. 2004; 20: 218-221.

11. Xie H, Zhang W, Cheng J, He Q. Laparoscopic versus open catheter placement in peritoneal dialysis patients: a systematic review and metaanalysis. BMC Nephrol. 2012; 13: 69.

12. Kavalakkat JP, Kumar S, Aswathaman K, Kekre NS. Continuous ambulatory peritoneal dialysis catheter placement: Is omentectomy necessary? Urol Ann. 2010; 2: 107-109. 